\title{
Diet and Exercise: a Match Made in Bone
}

\author{
Hubertine M.E. Willems ${ }^{1}$ - Ellen G.H.M. van den Heuvel $^{2}$ • Ruud J.W. Schoemaker ${ }^{2}$ • \\ Jenneke Klein-Nulend ${ }^{3} \cdot$ Astrid D. Bakker $^{3}$
}

Published online: 2 November 2017

(C) The Author(s) 2017. This article is an open access publication

\begin{abstract}
Purpose of Review Multiple dietary components have the potential to positively affect bone mineral density in early life and reduce loss of bone mass with aging. In addition, regular weight-bearing physical activity has a strong positive effect on bone through activation of osteocyte signaling. We will explore possible synergistic effects of dietary components and mechanical stimuli for bone health by identifying dietary components that have the potential to alter the response of osteocytes to mechanical loading.

Recent Findings Several (sub)cellular aspects of osteocytes determine their signaling towards osteoblasts and osteoclasts in response to mechanical stimuli, such as the osteocyte cytoskeleton, estrogen receptor $\alpha$, the vitamin D receptor, and the architecture of the lacunocanalicular system. Potential modulators of these features include 1,25-dihydroxy vitamin $\mathrm{D}_{3}$, several forms of vitamin $\mathrm{K}$, and the phytoestrogen genistein.

Summary Multiple dietary components potentially affect osteocyte function and therefore may have a synergistic effect on bone health when combined with a regime of physical activity.
\end{abstract}

This article is part of the Topical Collection on Osteocytes

Astrid D. Bakker

a.bakker@acta.nl

1 Department of Preventive Dentistry, Academic Center for Dentistry Amsterdam, Vrije Universiteit Amsterdam and University of Amsterdam, Amsterdam, The Netherlands

2 FrieslandCampina, Amersfoort, The Netherlands

3 Department of Oral Cell Biology, Academic Center for Dentistry Amsterdam, Vrije Universiteit Amsterdam and University of Amsterdam, Amsterdam Movement Sciences, Gustav Mahlerlaan 3004, 1081 LA Amsterdam, The Netherlands
Keywords Dietary components $\cdot$ Nutrition $\cdot$ Diet . Osteocytes $\cdot$ Bone health $\cdot$ Physical activity

\section{Introduction}

The incidence of osteoporosis-related fractures is expected to rise substantially over the coming decades [1]. It has been estimated that the annual number of fractures in the European Union will rise from 3.5 million in 2010 to 4.5 million in 2025, with a corresponding increase in costs, which was estimated at $€ 37$ billion in 2010 , including pharmacological intervention [2]. More importantly, osteoporosis-related fractures often lead to a diminished quality of life, disability, discomfort, and even death [2].

One safe way to prevent fractures is to positively affect bone mass and strength through mechanical stimuli. Lack of physical activity, e.g., as is seen in bedridden patients, results in a lack of mechanical stimuli and a rapid and substantial loss of bone mass [3]. High impact physical activity, on the other hand, has an anabolic effect on bone mineral content (BMC) and bone mineral density (BMD) [4]. The BMC and BMD have been shown to increase up to $20 \%$ in the loaded bone regions in athletes $[5,6]$. In general, high magnitude mechanical loads as well as loads applied at high frequency are known to increase bone mass, while low impact activities have less effect on bone, even when applied for a longer duration $[7,8 \bullet \bullet$. Unfortunately, exercise at a high intensity might be difficult for those elderly at risk of fractures, and the key may lie at building a sufficient "reserve" of bone mass at a young age. Indeed, bone mass at the age of 70 largely depends on peak bone mass reached before the age of 30 [9]. During growth, not only high impact activity but also low impact activity contributes to skeletal health [10-12]. 
High impact physical activity in childhood, especially when initiated before puberty, results in increased bone width and increased bone mineral content in girls and adolescent females [13]. It is much debated whether the efficiency of physical stimuli for increasing bone strength diminishes with age, especially after the menopause. Exercise probably does benefit bone health in adults since bone resorption and bone turnover markers are reduced by 1 month of moderate intensity exercise, i.e., four times per week and $30 \mathrm{~min}$ per day, in both pre- and postmenopausal women [14]. This suggests that mechanical loading of bones through exercise benefits bone mass at a young age as well as at a later age.

More than a century ago, Roux (1850-1924) proposed a concept in which mechanical loading of bone leads to the cell-mediated adaptation of bone structure, resulting in an optimized load-bearing capacity of bone [15]. It then took several decades until the groundbreaking experiments by the groups of Nijweide and Burger in the Netherlands identified osteocytes as the chief mechanosensing cells in bone $[16,17]$. Definite proof that osteocytes sense mechanical signals and respond by directing osteoclast activity came from animal experiments performed by Tatsumi et al., demonstrating a lack of bone loss in unloaded hind limbs of mice missing the majority of their osteocytes [18]. Activation of osteocyte signaling through exercise thus provides a way to prevent osteoporosis by maintaining, or even enhancing, bone mass.

Besides daily physical activity, a healthy diet is among the most commonly advocated lifestyle measures to improve (skeletal) health [19]. Recently, the National Osteoporosis Foundation wrote a position statement on peak bone mass development. The best evidence (grade A) is available for positive effects of calcium intake and physical activity, especially during the late childhood and peripubertal years - a critical period for bone accretion [19]. "European guidance for the diagnosis and management of osteoporosis in postmenopausal women" recommends a daily intake of at least $1000 \mathrm{mg} /$ day for calcium, $800 \mathrm{IU} /$ day for vitamin D, and $1 \mathrm{~g} / \mathrm{kg}$ body weight of protein for all women aged over 50 years [20]. Persons need to consume an overall healthy diet, like increasing plant-based foods or dairy foods, to meet their nutrient requirements [21].

The aim of this review is to identify potential interactions between dietary components and mechanical stimuli with respect to their effect on osteocytes and bone health. To this end, we defined cellular aspects of osteocytes that determine their signaling towards osteoblasts and osteoclasts in response to mechanical stimuli (Fig. 1) and by describing a number of dietary components with the ability to affect these cellular structures, thereby potentially enhancing the response of osteocytes to mechanical loading.
Genetic and epigenetic properties

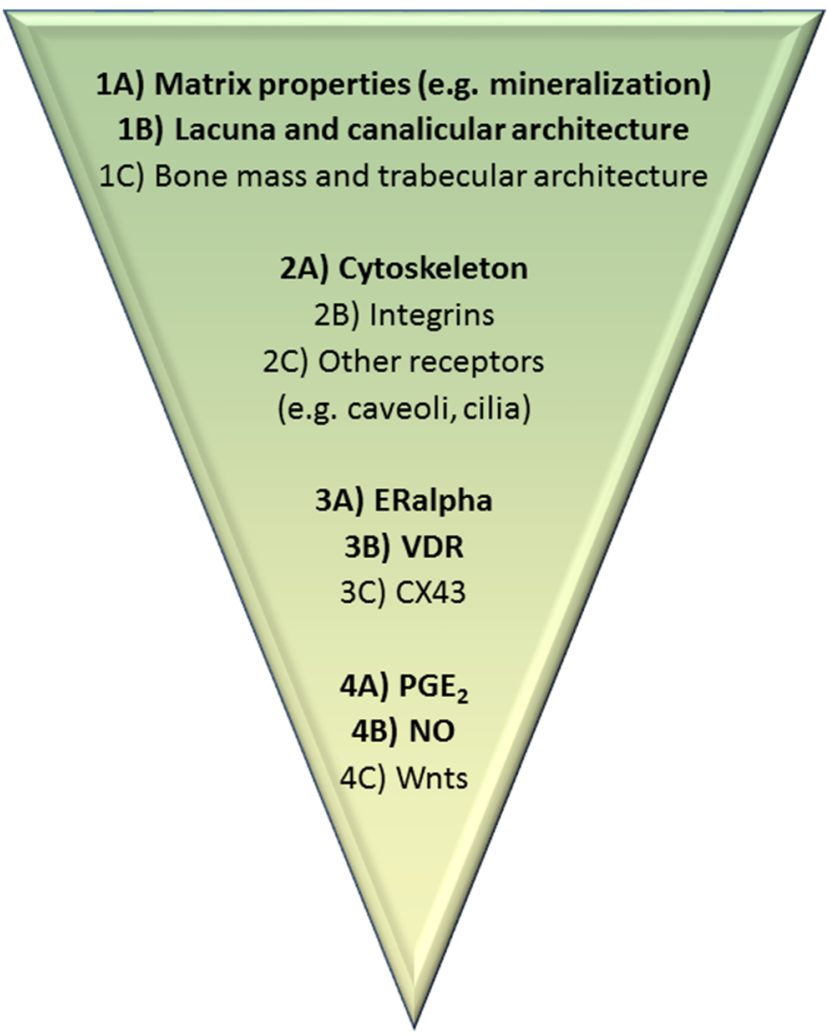

Fig. 1 Mechanotransduction. Physical mechanical loads elicit a biological response through the process of mechanotransduction. For bone, this process can be broken down in the following steps: (1) Transmission of the bulk mechanical stimulus to the osteocyte, (2) Sensing of the mechanical stimulus by osteocytes and transduction into a chemical response, (3) Modulation of intracellular signaling, (4) Production of signaling molecules. The last step leads to an altered osteoclast and osteoblast recruitment and activity, and an alteration in bone mass and structure. Changes on each of these levels of mechanotransduction, for example, by affecting the cellular structures described in the figure, will potentially affect the efficacy of mechanical stimuli for modulating bone mass and structure, and thereby the ability of bone to withstand fracture. Governing all steps of the process of mechanotransduction is the genetic make-up of the osteocytes

\section{The Combined Effect of Nutrition and Mechanical Loading on Bone In Vivo}

Since mechanical loading is beneficial for bone mass and nutrition affects bone mass, the question then rises what is known about the combination of exercise and diet with respect to bone mass in animals and humans. It is difficult to give a clear answer, as positive effects, negative effects, and a lack of effects have been reported regarding the effect of exercise and dietary changes on bone mass. To provide a few examples, in adult male rats, bone morphology and strength are non-significantly affected by $30 \%$ food restriction combined with voluntary exercise training after a 13-week experimental period [22]. In young female rats, on the other hand, food restriction in combination 
with voluntary exercise significantly reduced bone strength, bone mineral density, and calcium absorption compared to an exercise group. This deficit could be partially rescued by calcium supplementation, suggested that calcium supplementation has a positive effect on bone strength in combination with exercise [23•]. On the other hand, dietary methionine restriction (MR) and endurance exercise down-regulate bone and energy metabolic indices, bone size, and extrinsic bone strength in growing rats, which appears to be part of an adaptive response to changes in energy metabolism in the entire organism. On a positive note, there is the possibility that MR and endurance exercise reduce bone aging by slowing bone turnover and enhancing energy metabolism [24].

In humans, a high intake of dairy, calcium, and protein during diet- and exercise-induced weight loss in premenopausal overweight and obese women favorably affects bone health biomarkers [25]. Inversely, low calcium intake diminishes the increased bone mass resulting from exercise in prepubertal girls [26]. One study conducted on the effect of diet and physical activity in healthy subjects (age 14-18 yrs.) shows that the main dietary variables related to bone mass are energy intake, calcium, vitamin D, and servings of dairy products, in combination with vigorous (jumping) physical activity [27]. Another study in well-trained female cyclists showed that a calcium-rich pre-exercise breakfast meal containing $\sim 1350 \mathrm{mg}$ of calcium consumed as compared to no calcium $\sim 90 \mathrm{~min}$ before a prolonged and high intensity bout of stationary cycling attenuates the exercise-induced rise in markers of bone resorption [28]. A 2-year study showed that exercise was effective in reducing fall-related injuries among community-dwelling older women at a moderate cost. Vitamin D supplementation had marginal additional benefit [29]. An observational study in young adult men indicated that habits of consuming breakfast and exercising at least $10 \mathrm{~h}$ per week during high school were linked with significantly higher L2-4 and femoral neck BMDs [30]. In osteoporotic sedentary women, an intervention with soy isolate protein or soy in combination with progressive resistance exercises four times/week for 12 weeks significantly stimulates bone and muscle strength gains. Interestingly, the improvements are more pronounced in the soy-and-exercise group [31]. The combination of improving nutrition (adequate energy and vitamin D) and resistance exercise during spaceflight attenuates the expected BMD deficits previously observed after 4- to 6month missions [32], showing that a proper combination of nutrition and exercise serves to maintain bone mass, even under extreme conditions.

The major drawbacks of studies on bone in vivo in animals and humans are the associated high costs and the long-term duration of the experiments. Moreover, the added benefit of a combination of physical activity and optimal dietary component status seems rather difficult to prove. However, it remains a tantalizing idea that osteoporosis can be prevented by providing the optimal combination of exercise and nutrition. A targeted approach to solving the question whether diet and exercise can synergistically benefit bone mass and strength is to start to answer the question whether it is possible to identify dietary components that enhance the response of osteocytes to mechanical stimuli. In multiple cases, the effects of dietary components on osteocytes have not been investigated, and effects on osteoblasts are described instead. Osteocytes are terminally differentiated osteoblasts, but care has to be taken when extrapolating results obtained with osteoblasts towards osteocytes, because they also have distinct differences in morphology and function as outlined below.

\section{Degree of Differentiation}

Osteocytes are derived from osteoblasts, and osteoblasts and osteoblastic cell lines show mechanosensitivity [33••]. When osteoblasts differentiate into osteocytes, they become more sensitive to mechanical loading [33••]. Thus, by stimulating the differentiation of osteoblasts into osteocytes, it may be possible to enhance the sensitivity of bone tissue to mechanical stimuli, and therefore dietary components that are able to stimulate osteoblast differentiation might ultimately lead to changes in bone mass. Several of such components exist. The component fluoride is well known for its effect on osteoblast differentiation. It increases osteoblast proliferation and differentiation in a rat osteosarcoma cell line [34]. Another component that enhances osteoblast differentiation is lactoferrin, a pleiotropic factor and well-known dairy ingredient [35]. Lactoferrin stimulates both osteoblast proliferation and differentiation into osteocytes [36]. Other components that stimulate osteoblast differentiation are phytoestrogens, such as genistein, daidzein, diarylheptanoid and 8prenylnaringenin $[37,38]$, and therefore it is possible that they affect the mechanoresponse of cells from the osteoblast lineage as well. Vitamin K2 inhibits miR 133a expression, which is accompanied by enhanced osteogenic differentiation of mesenchymal stem cells, but whether vitamin $\mathrm{K}$ also enhances the differentiation of osteoblasts towards osteocytes remains to be determined [39]. Additional components known to enhance osteoblast differentiation are strontium, isoflavones, and whey protein [40-42]. Whether any of these dietary components actually leads to an increase in the anabolic response of bone tissue - as a whole - to mechanical loading remains to be investigated. The increase in mechanosensitivity of terminally differentiated osteoblasts is likely related to the rigorous changes in the cytoskeleton associated with the transition of an osteoblast into an osteocyte [43]. How osteocyte mechanosensitivity and the cytoskeleton are related, and how this could be affected by dietary components, is elucidated next. 


\section{Cytoskeleton}

Lately, the view of the cytoskeleton as a structure responsive to external physical and chemical stimuli has become prominent [44]. The cytoskeleton is involved in mechanosensing and is a key determinant of the material properties of the cell. Osteocyte morphology, determined by the cytoskeleton, is correlated to the magnitude of the response to mechanical stimuli, whereby round osteocytes seem to have a higher response to mechanical stimuli than more spread, adherent osteocytes [45]. The shape of cytoskeletal structures depends on the dynamics of actin fibers, intermediate filaments, and microtubules [46, 47]. Actin fibers are most abundant in eukaryotic cells and form a framework that supports and shapes the plasma membrane. Intermediate filaments are relatively under investigated in mesenchymal cells, although they play an important role in the resistance of cells against shear forces. Microtubules exhibit structural and functional polarity and are important components of primary cilia [48], a mechanosensitive structure that is present on osteocytes [49].

When thinking of cytoskeleton-affecting dietary components, a few come to mind. First, fluoride is known to disrupt the actin cytoskeleton of protozoa [50] and actin fibers in ameloblasts [51], leading to a disrupted actin cytoskeleton and more rounded cells [51]. Fluoride might thus enhance osteocyte mechanosensitivity by altering osteocyte shape, although the intake would have to be relatively high, considering the dose required to affect the actin cytoskeleton. Second, oleuropein comes to mind. Osteoporosis-related fractures are lowest in Southern Europe, which is likely related to dietary influences [52]. Olive oil and its main compound oleuropein are abundantly present in the Mediterranean diet. The polyphenol oleuropein disrupts microtubules in tumor cells, also resulting in rounded cells and altered cytoskeletal organization [53]. Third, genistein, an isoflavone, and phytoestrogen mostly present in soybeans depolymerizes the microtubules in human A549 epithelium cancer cells and inhibits microtubule polymerization in vitro [54]. One can hypothesize that oleuropein and genistein affect osteocyte shape and primary cilium formation, thereby altering the sensitivity of osteocytes to mechanical loading and affecting bone mass. These findings allow for a hypothetical role for fluoride, oleuropein, and genistein in the mechanoresponse of osteocytes, making them an interesting target for future investigations.

\section{Estrogen Receptor Alpha}

Estrogen is known to have profound bone preserving effects. Decreased estrogen levels following menopause have a strong negative effect on bone mass [55]. On the other hand, administration of exogenous estrogen increases bone mineral density in humans and seems especially beneficial when combined with an exercise regime [56, 57]. Estrogen can affect bone resorption and bone formation after binding to its receptors present on both osteoclasts and osteoblasts, the net effect depending on the type of receptor (i.e., estrogen receptor (ER) $\alpha$ or $\beta$ ) being activated $[55,58]$. In addition, estrogen likely alters the response of osteocytes to mechanical loading via the estrogen receptor $\alpha$ and ER $\beta$ [59••, 60], thereby affecting bone mass. Mechanical loading is able to preserve osteocyte and osteoblast viability, because $\operatorname{ER} \alpha$, as well as $\operatorname{ER} \beta$, activates ERK. Interestingly, the ligand-binding domain of each receptor suffices for mechanosensation, in a ligandindependent fashion, and both plasma membrane localization of the ER $\alpha$ and its interaction with caveolin- 1 are required for mechanotransduction [60]. It has been suggested that osteocytes become less sensitive to mechanical stimuli in woman after menopause due to the estrogen loss and alterations in $\mathrm{ER} \alpha$ expression, thereby explaining the rapid loss in bone mass associated with menopause [61, 62]. Phytoestrogens are plant-derived compounds with estrogen-like activity, and supplementation with phytoestrogens likely prevents the reduction in BMD associated with menopause and maintains a healthy bone structure [63]. Little is known about the ability of phytoestrogens to modulate ER $\alpha$ expression, but at least genistein enhances ER $\alpha$ expression in MC3T3-E1 mouse osteoblasts [64], suggesting that genistein, similar to endogenous estrogens, is able to modulate ER $\alpha$ expression, and thus potentially alter osteocyte mechanosensitivity.

\section{Vitamin D Receptor}

Not only ER $\alpha$ but also the vitamin D receptor (VDR) is expressed by osteocytes and has been linked to responses of bone cells to mechanical stimuli. The VDR-mediated genomic actions of 1,25 dihydroxy vitamin $\mathrm{D}_{3}$ (hereafter referred to as "vitamin D") occur by coupling of the VDR to VDR response elements (VDRE) in the promoter regions of vitamin $\mathrm{D}$ target genes [65]. Genes containing a VDRE often encode for proteins involved in the regulation of osteoclast formation and/or activity, or osteoblast differentiation, such as receptor activator of nuclear factor kappa- $\beta$ ligand (RANKL) or nitric oxide synthase, the enzyme responsible for nitric oxide (NO) production, e.g., in response to mechanical loading [66]. This prompts the hypothesis that vitamin $\mathrm{D}$ enhances the production of signaling molecules by mechanically stimulated osteocytes via genomic actions of the VDR. However, mechanical loading of osteoblasts in vitro through a controlled pulsating fluid flow (PFF) rapidly increases NO production in osteoblasts, but this PFF effect is abolished, rather than enhanced, after $24 \mathrm{~h}$ of vitamin D preincubation [67]. Vitamin D may affect mechanical loading-induced NO production independent of genomic VDR action, since it diminishes PFFinduced NO production in osteoblasts derived from genetically altered mice that lack the ability for genomic VDR action 
[67]. Vitamin D and mechanical loading thus interact at the level of mechanotransduction, at least in vitro.

The role of vitamin D in bone mineral homeostasis is to promote intestinal absorption of calcium and phosphate [68]. Vitamin D regulates calcium and phosphate homeostasis through cross-regulation of PTH, but also via osteoclastogenesis and osteoblastogenesis, calcium and phosphate acquisition, and regulation of anabolic and catabolic gene expression to achieve a proper mineral and skeletal metabolism [69]. Vitamin D deficiency results in increased PTH levels, and PTH reduces the mechanical stress-induced NO production in human primary bone cells in vitro, suggesting that vitamin D might affect the response of osteocytes to mechanical loading via PTH [70]. Taken together, vitamin D and mechanical loading are likely to interact, either via the VDR or via modulation of PTH levels.

\section{Lacunocanalicular Network Architecture and Bone Matrix}

PTH may directly affect the response of bone cells to mechanical stimulation, but it may also affect the perception of mechanical stimuli in vivo. Continuously elevated levels of PTH cause osteocytic osteolysis [71], thereby affecting the architecture of the lacunacanalicular system that forms the niche for the osteocytes embedded within the bone matrix. Altered lacunar shape alters the quantity of local strains occurring around the osteocytes during daily activities and exercise, as outlined elsewhere in this issue, thereby effectively altering the height of the mechanical stimulus experienced by osteocytes [72]. Thus, dietary components that alter osteocyte lacunar shape will most likely interact with mechanotransduction by osteocytes. Regarding matrix strains transduced to osteocytes, dietary components that significantly alter the mineralization of the bone matrix, thereby rendering it more stiff or compliant, will theoretically affect the amount of strain elicited on osteocytes. Dietary components that significantly alter the mineralization of the bone matrix could also affect the ability of osteocytes to maintain an unmineralized layer of matrix surrounding their cell fingers. It is generally assumed that this layer is essential for the transmission of mechanical signals, since loading-induced shifts in interstitial fluid through the canaliculi wherein the osteocyte cell extensions reside is considered an important amplification mechanism for mechanical signals exerted on bone [17]. Factors produced by osteoblasts and osteocytes that affect mineralization include osteocalcin (OC) and matrix GLA protein (MGP). MGP has been found in bone, dentine, cartilage, and soft tissue, including blood vessels, and is associated with the organic matrix and mobilization of calcium. Animal studies show that MGP prevents the calcification of soft tissue and cartilage while facilitating normal bone growth and development. The synthesis of OC and MGP is regulated by calcitriol, retinoic acid, vitamin $\mathrm{K}$, and vitamin D [73]. In addition, it was shown that feeding rats olive extract at $250 \mathrm{mg}$ per day for 12 months increases serum osteocalcin [74]. Whether this also affects matrix mineralization and transmission of mechanical loads towards the osteocytes is unknown. Matrix mineralization is definitely affected by retinol intake in mice, at least under extreme conditions of disturbed matrix mineralization [75]. X-linked hypophosphatemic rickets is caused by inactivation of the PHEX gene. Phex is expressed by very late differentiated osteoblasts and early osteocytes in mice and humans, while it is exclusively expressed by osteocytes in chickens [16]. Feeding mice with a mutant PHEX gene, a retinol-free diet results in a partial rescue of growth plate and bone mineralization defects, while the amount of non-mineralized bone matrix is reduced more than $70 \%$, showing the impact of retinol on the cellautonomous mineralization defect of Phex-deficient osteoblasts [75]. Whether retinol in the diet will have a similar effect on matrix mineralization in otherwise healthy humans remains to be seen. Taken together, vitamin D may affect the transmission of mechanical signals to osteocytes if it affects lacuna shape, but whether any other dietary component will affect transmission of mechanical signals is uncertain.

\section{Signaling Molecule Production}

Osteocytes produce soluble factors such as nitric oxide (NO), prostaglandin $\mathrm{E}_{2}\left(\mathrm{PGE}_{2}\right)$, bone morphogenic proteins (BMPs), Wnts, sclerostin, RANKL, and osteoprotegerin (OPG) in response to changes in mechanical loading. If a dietary component can alter the production of these signaling molecules by osteocytes in response to mechanical loading, it has the potential to enhance bone mass in concert with exercise.

$\mathrm{NO}$ is a well-known early mediator of bone formation and is essential for the anabolic response of bone to mechanical loading in vivo [76]. NO also is an essential mediator for the reduced stimulation of osteoclast formation by mechanically loaded osteocytes compared to unloaded osteocytes [77]. Both animal studies and studies in humans support the use of NO donors to prevent bone loss [78]. Since NO is formed during the conversion of L-arginine by NOS, it is feasible that the amino acid arginine plays a role in the process of adaptive bone formation. Several in vitro studies have shown that arginine administration significantly increases NO production, as well as alkaline phosphatase and insulin-like growth factor-I production, and type I collagen synthesis by human osteoblasts and in osteoblasts derived from calvariae of newborn rats [79]. Low concentrations of oleuropein $\left(10^{-4}-10^{-6} \mathrm{M}\right)$ increase NO production via induction of inducible NOS by 
Table 1 Summary of dietary components potentially able to affect osteocyte properties

Arginine Calcitriol Lactoferrin Daidzein Fluoride Genistein Strontium Oleuropein Retinoic acid Vitamin D Vitamin K

\begin{tabular}{|c|c|c|c|c|c|c|c|c|c|c|c|}
\hline Lacuna shape & & & & & & & & & & $+/-$ & \\
\hline Matrix mineralization & & + & & & & & & & + & + & + \\
\hline Cytoskeletal changes & & & & & + & + & & + & & & \\
\hline ER $\alpha$ expression & & & & + & & + & & & & & \\
\hline VDR signaling & & & & & & & & & & + & \\
\hline Osteogenic diff. & & & + & + & + & + & + & & & + & + \\
\hline Signal molecules & + & & & & & & + & + & & + & + \\
\hline
\end{tabular}

macrophages in a mouse infection model, thus increasing functional activity of these cells [80]. Both these dietary components could thus have a beneficial effect on NO production by mechanically stimulated osteocytes.

Prostaglandins are generated by the release of arachidonic acid from phospholipids in the cell membrane, followed by conversion of arachidonic acid into prostaglandin G2 and subsequently prostaglandin $\mathrm{H} 2$ by $\mathrm{COX}$ [81]. Prostaglandin $\mathrm{H} 2$ is further isomerized to the biological active prostanoids, such as $\mathrm{PGE}_{2}$ [81]. Oleuropein at 10-100 $\mu \mathrm{g} / \mathrm{ml}$ reduces the levels of COX-2 seen in inflammation [82]. Another polyphenolic compound, chlorogenic acid, decreases inflammationinduced production of $\mathrm{PGE}_{2}$ as well as the expression of COX-2 in RAW 264.7 macrophages [83], indicating a role for polyphenols in inflammation-induced bone resorption. Finally, strontium is known for its effect on the proliferation of pre-osteoblasts and the stimulation of bone formation [84]. In both MC3T3-E1 osteoblasts and MLO-Y4 mouse osteocyte-like cells, strontium stimulates the production of $\mathrm{PGE}_{2}$ [85]. Whether oleuropein or chlorogenic acid increases the response of osteocytes to mechanical loading is unknown. Strontium does not seem to have a synergistic effect on the production of $\mathrm{PGE}_{2}$ by mechanically loaded osteocytes in vitro, but it does have an additive effect [85].

RANKL is a membrane-bound cytokine and binds to its receptor RANK on osteoclast precursors [86•]. Osteocyte RANKL is a critical mediator of bone loss in response to calcium deficiency [86•]. The severe osteopetrotic (abnormal high bone density) phenotype observed in adult mice specifically lacking osteocyte-derived RANKL indicates that osteocytes are the major source of RANKL during bone remodeling in vivo thereby determining bone mass [87]. Binding of osteoprotegerin (OPG) to RANK results in suppression of osteoclast formation in vivo and in vitro [88]. The ratio OPG/RANKL is therefore considered crucial in osteoclast formation [89]. RANKL is also expressed by osteocytes during bone microdamage [90], and apoptotic osteocytes initiate bone resorption by recruitment of osteoclast precursor cells to the local damage site via RANKL production [91••].
Mechanical loading enhances OPG production and reduces RANKL production by osteocytes. This effect of mechanical loading on RANKL production may be modified via the intake of components that are known to affect RANKL production such as vitamin K. Vitamin $\mathrm{K}$ is present as phylloquinone (vitamin K1) and menaquinones (vitamin K2). Examples of the latter are MK-7, MK-8, and MK-9 [92]. Vitamin K2 inhibits osteoclast formation by decreasing RANKL [93]. MK-7 suppresses proliferation, but enhances OPG, RANKL, and RANK gene and protein expression in MC3T3-E1 osteoblasts $[94,95]$. This opens the possibility that vitamin $\mathrm{K}$ affects the mechanical loading-mediated communication between osteoblasts and osteoclasts. There are many other signaling molecules produced by osteocytes in response to mechanical (un)loading of bone, many of which can likely be affected by dietary components in one way or the other. In this review, we will restrict the discussion to aforementioned signaling molecules as they have been the most extensively studied.

\section{Conclusions}

The increasing number of patients suffering from osteoporosis is accompanied by high costs and decreased quality of life, and therefore new curative and therapeutic approaches are urgently needed. Given the crucial importance of dietary components and physical activity for bone health in general, much is likely to be gained from a regime of daily exercise and a balanced diet. However, more research is needed to obtain a better understanding of the ability of osteocytes to respond to mechanical stimuli in patients with osteoporosis as this might be altered compared to others without osteoporosis [96]. More research is also urgently required to gain a better understanding of the molecular mechanisms involved in the process of mechanotransduction in osteocytes to aid the development of optimal bone-anabolic treatments. In this review, several mechanisms have been identified by which dietary components potentially modulate the beneficial effect of mechanical stimuli on bone health. This review also 
identified a number of dietary components, e.g., fluoride, oleuropein, (phyto)estrogens, lactoferrin, strontium, vitamin $\mathrm{K}$, and vitamin $\mathrm{D}$ with potential for enhancing bone health when applied in combination with mechanical stimuli (Table 1). This list can likely be expanded in the future as research progresses, holding great promise for finding a balanced curative or therapeutic approach for the prevention of age-related bone loss.

\section{Compliance with Ethical Standards}

Conflict of Interest Ellen GHM van den Heuvel and Ruud JW Schoemaker are employees of FrieslandCampina, a dairy company.

Astrid Bakker, Jenneke Klein-Nulend, and Hubertine Willems declare no conflict of interest.

Human and Animal Rights and Informed Consent This article does not contain any studies with human or animal subjects performed by any of the authors.

Open Access This article is distributed under the terms of the Creative Commons Attribution 4.0 International License (http:// creativecommons.org/licenses/by/4.0/), which permits unrestricted use, distribution, and reproduction in any medium, provided you give appropriate credit to the original author(s) and the source, provide a link to the Creative Commons license, and indicate if changes were made.

\section{References}

Papers of particular interest, published recently, have been highlighted as:

- Of importance

•- Of major importance

1. Brewer L, Williams D, Moore A. Current and future treatment options in osteoporosis. Eur J Clin Pharmacol. 2011;67:321-31. https://doi.org/10.1007/s00228-011-0999-2.

2. Hernlund E, Svedbom A, Ivergard M, Compston J, et al. Osteoporosis in the European Union: medical management, epidemiology and economic burden. A report prepared in collaboration with the International Osteoporosis Foundation (IOF) and the European Federation of Pharmaceutical Industry Associations (EFPIA). Arch Osteoporos. 2013;8:136. https://doi.org/10.1007/ s11657-013-0136-1.

3. Jiang S, Jiang L, Dai L. Mechanisms of osteoporosis in spinal cord injury. Clin Endocrinol. 2006;65:555-65. https://doi.org/10.1111/j. 1365-2265.2006.02683.x.

4. Hind K, Burrows M. Weight-bearing exercise and bone mineral accrual in children and adolescents: a review of controlled trials. Bone. 2007;40:14-27. https://doi.org/10.1016/j.bone.2006.07.006.

5. Karlsson MK, Linden C, Karlsson C, et al. Exercise during growth and bone mineral density and fractures in old age. Lancet. 2000;355:469-70.

6. Karlsson MK, Magnusson H, Karlsson C, et al. The duration of exercise as a regulator of bone mass. Bone. 2001;28:128-32.

7. Zinner C, Baessler B, Weiss K, Ruf J, Michels G, Holmberg HC, et al. Effect of resistance training with vibration and compression on the formation of muscle and bone. Muscle Nerve. 2017; https://doi. org/10.1002/mus. 25560 .
8.• Gomez-Bruton A, Montero-Marín J, González-Agüero A, GómezCabello A, García-Campayo J, Moreno LA, et al. Swimming and peak bone mineral density: a systematic review and meta-analysis. J Sports Sci. 2017; https://doi.org/10.1080/02640414.2017.1307440. This paper systematically reviewed recent papers and shows that swimming does not have a beneficial effect on bone mass.

9. Hui SL, Slemenda CW, Johnston CC Jr. The contribution of bone loss to postmenopausal osteoporosis. Osteoporos Int. 1990;1:30-4.

10. MacKelvie KJ, Petit MA, Khan KM, et al. Bone mass and structure are enhanced following a 2-year randomized controlled trial of exercise in prepubertal boys. Bone. 2004;34:755-64. https://doi.org/ 10.1016/j.bone.2003.12.017.

11. Löfgren B, Detter F, Dencker M, Stenevi-Lundgren S, Nilsson JÅ, Karlsson MK. Influence of a 3-year exercise intervention program on fracture risk, bone mass, and bone size in prepubertal children. $\mathrm{J}$ Bone Miner Res. 2011;26:1740-7. https://doi.org/10.1002/jbmr. 381.

12. Laing EM, Wilson AR, Modlesky CM, et al. Initial years of recreational artistic gymnastics training improves lumbar spine bone mineral accrual in 4- to 8-year-old females. J Bone Miner Res. 2005;20:509-19. https://doi.org/10.1359/JBMR.041127.

13. Hasselstrom HA, Karlsson MK, Hansen SE, et al. A 3-year physical activity intervention program increases the gain in bone mineral and bone width in prepubertal girls but not boys: the prospective Copenhagen School Child Interventions Study (CoSCIS). Calcif Tissue Int. 2008;83:243-50. https://doi.org/10.1007/s00223-0089166-x.

14. Kitareewan W, Boonhong J, Janchai S, et al. Effects of the treadmill walking exercise on the biochemical bone markers. J Med Assoc Thail. 2011;94:S10-6.

15. Lee TC, Taylor D. Bone remodeling: should we cry Wolff? Ir Med Sci. 1999;168:102-5.

16. van der Plas A, Aarden EM, Feijen JH, de Boer AH, Wiltink A, Alblas MJ, et al. Characteristics and properties of osteocytes in culture. J Bone Miner Res. 1994;9:1697-704. https://doi.org/10. 1002/jbmr.5650091105.

17. Klein-Nulend J, van der Plas A, Semeins CM, Ajubi NE, Frangos JA, Nijweide PJ, et al. Sensitivity of osteocytes to biomechanical stress in vitro. FASEB J. 1995;9:441-5.

18. Tatsumi S, Ishii K, Amizuka N, Li M, Kobayashi T, Kohno K, et al. Targeted ablation of osteocytes induces osteoporosis with defective mechanotransduction. Cell Metab. 2007;5:464-75. https://doi.org/ 10.1016/j.cmet.2007.05.001.

19. Weaver CM, Gordon CM, Janz KF, et al. The National Osteoporosis Foundation's position statement on peak bone mass development and lifestyle factors: a systematic review and implementation recommendations. Osteoporos Int. 2016;27:1281-386. https://doi.org/10.1007/s00198-015-3440-3.

20. Rizzoli R, Stevenson JC, Bauer JM, et al. The role of dietary protein and vitamin $\mathrm{D}$ in maintaining musculoskeletal health in postmenopausal women: a consensus statement from the European Society for Clinical and Economic Aspects of Osteoporosis and Osteoarthritis (ESCEO). Maturitas. 2014;79:122-32. https://doi. org/10.1016/j.maturitas.2014.07.005.

21. Cifelli CJ, Houchins JA, Demmer E, et al. Increasing plant based goods or dairy foods differentially affects nutrient intakes: dietary scenarios using NHANES 2007-2010. Nutrients. 2016;8 https:// doi.org/10.3390/nu8070422.

22. Hattori S, Park JH, Agata U, Akimoto T, Oda M, Higano M, et al. Influence of food restriction combined with voluntary running on bone morphology and strength in male rats. Calcif Tissue Int. 2013;93:540-8. https://doi.org/10.1007/s00223-013-9787-6.

23. Aikawa Y, Agata U, Kakutani Y, Kato S, Noma Y, Hattori S, et al. The preventive effect of calcium supplementation on weak bones caused by the interaction of exercise and food restriction in young female rats during the period from acquiring bone mass to 
maintaining bone mass. Calcif Tissue Int. 2016;98:94-103. This paper shows the importance of a calcium in the diet, especially in young physically active animals.

24. Huang TH, Lewis JL, Lin HS, Kuo LT, Mao SW, Tai YS, et al. A methionine-restricted diet and endurance exercise decrease bone mass and extrinsic strength but increase intrinsic strength in growing male rats. J Nutr. 2014;144:621-30.

25. Josse AR, Atkinson SA, Tarnopolsky MA, et al. Diets higher in dairy foods and dietary protein support bone health during diet- and exercise-induced weight loss in overweight and obese premenopausal women. J Clin Endocrinol Metab. 2012;97:251-60.

26. Ondrak KS, Morgan DW. Physical activity, calcium intake and bone health in children and adolescents. Sports Med. 2007;37: 587-600.

27. Rowlands AV, Ingledew DK, Powell SM, et al. Interactive effects of habitual physical activity and calcium intake on bone density in boys and girls. J Appl Physiol. 2004;97:1203-8.

28. Haakonssen EC, Ross ML, Knight EJ, et al. The effects of a calcium-rich pre-exercise meal on biomarkers of calcium homeostasis in competitive female cyclists: a randomised crossover trial. PLoS One. 2015;10:e0123302. https://doi.org/10.1371/journal. pone.0123302.

29. Patil R, Kolu P, Raitanen J, et al. Cost-effectiveness of vitamin D supplementation and exercise in preventing injurious falls among older home-dwelling women: findings from an RCT. Osteoporos Int. 2016;27:193-201. https://doi.org/10.1007/s00198-015-3240-9.

30. Ishimoto Y, Yoshida M, Nagata K, Yamada H, Hashizume H, Yoshimura N. Consuming breakfast and exercising longer during high school increases bone mineral density in young adult men. J Bone Miner Metab. 2013;31:329-36. https://doi.org/10.1007/ s00774-012-0415-8.

31. Shenoy S, Bedi R, Sandhu JS. Effect of soy isolate protein and resistance exercises on muscle performance and bone health of osteopenic/osteoporotic post-menopausal women. J Women Aging. 2013;25:183-98. https://doi.org/10.1080/08952841.2013. 764252.

32. Smith SM, Heer MA, Shackelford LC, Sibonga JD, Ploutz-Snyder L, Zwart SR. Benefits for bone from resistance exercise and nutrition in long-duration spaceflight: evidence from biochemistry and densitometry. J Bone Miner Res. 2012;27:1896-906. https://doi. org/10.1002/jbmr.1647.

33.• Suswillo RF, Javaheri B, Rawlinson SC, Dowthwaite GP, Lanyon LE, Pitsillides AA. Strain uses gap junctions to reverse stimulation of osteoblast proliferation by osteocytes. Cell Biochem Funct. 2017;35:56-65. https://doi.org/10.1002/cbf.3245. Paper showing convincing evidence of osteocyte to osteoblast communication in vitro in the absence and presence of mechanical stimuli.

34. Qu H, Wie M. The effect of fluoride contents in fluoridated hydroxyapatite on osteoblast behavior. Acta Biomater. 2006;2:113-9.

35. Naot D, Grey A, Reid IR, et al. Lactoferrin - a novel bone growth factor. Clin Med Res. 2005;3:93-101.

36. Takayama Y, Mizumachi K. Effect of lactoferrin-embedded collagen membrane on osteogenic differentiation of human osteoblastlike cells. J Biosci Bioeng. 2009;107:191-5.

37. Bhukhai K, Suksen K, Bhummaphan N, Janjorn K, Thongon N, Tantikanlayaporn D, et al. A phytoestrogen diarylheptanoid mediates estrogen receptor/Akt/glycogen synthase kinase $3 \beta$ proteindependent activation of the $\mathrm{Wnt} / \beta$-catenin signaling pathway. $\mathrm{J}$ Biol Chem. 2012;287:36168-78. https://doi.org/10.1074/jbc. M112.344747.

38. Luo D, Kang L, Ma Y, Chen H, Kuang H, Huang Q, et al. Effects and mechanisms of 8-prenylnaringenin on osteoblast MC3T3-E1 and osteoclast-like cells RAW264.7. Food Sci Nutr. 2014;2:34150. https://doi.org/10.1002/fsn3.109.

39. Zhang Y, Weng S, Yin J, Ding H, Zhang C, Gao Y. Vitamin K2 promotes mesenchymal stem cell differentiation by inhibiting miR 133a expression. Mol Med Rep. 2017; https://doi.org/10.3892/ mmr.2017.6308

40. Sila-Asna M, Bunyaratvej A, Maeda S, et al. Osteoblast differentiation and bone formation gene expression in strontium-inducing bone marrow mesenchymal stem cell. Kobe J Med Sci. 2007;53: 25-35.

41. Pan W, Quarles LD, Song LH, et al. Genistein stimulates the osteoblastic differentiation via NO/cGMP in bone marrow culture. J Cell Biochem. 2005;94:307-16.

42. Takada Y, Aoe S, Kumegawa M. Whey protein stimulated the proliferation and differentiation of osteoblastic MC3T3-E1 cells. Biochem Biophys Res Commun. 1996;14:445-9.

43. Tanaka-Kamioka K, Kamioka H, Ris H, Lim SS. Osteocyte shape is dependent on actin filaments and osteocyte processes are unique actin-rich projections. J Bone Miner Res. 1998;13:1555-68.

44. Klein-Nulend J, Bacabac RG, Bakker AD. Mechanical loading and how it affects bone cells: the role of the osteocyte cytoskeleton in maintaining our skeleton. Eur Cell Mater. 2012;24:278-91.

45. Bacabac RG, Mizuno D, Schmidt CF, et al. Round versus flat: bone cell morphology, elasticity, and mechanosensing. J Biomech. 2008;41:1590-8.

46. Bradke F, Dotti CG. The role of local actin instability in axon formation. Science. 1999;283:1931-4.

47. Kunda P, Paglini G, Quiroga S, et al. Evidence for the involvement of Tiam1 in axon formation. J Neurosci. 2001;21:2361-72.

48. Lee KL, Guevarra MD, Nguyen AM, Chua MC, Wang Y, Jacobs $\mathrm{CR}$. The primary cilium functions as a mechanical and calcium signaling nexus. Cilia. 2015;29:7.

49. Whitfield JF. Primary cilium - is it an osteocyte's strain-sensing flowmeter? J Cell Biochem. 2003;89:233-7.

50. Kovács P, Pállinger E, Csaba G. Effects of sodium fluoride (NaF) on the cilia and microtubular system of Tetrahymena. Cell Biochem Funct. 2008;26:591-7.

51. Li Y, Decker S, Yuan ZA, et al. Effects of sodium fluoride on the actin cytoskeleton of murine ameloblasts. Arch Oral Biol. 2005;50: 681-8.

52. Orchard TS, Pan X, Cheek F, et al. A systematic review of omega-3 fatty acids and osteoporosis. Br J Nutr. 2012;107:S253-60.

53. Hamdi HK, Castellon R. Oleuropein, a non-toxic olive iridoid, is an anti-tumor agent and cytoskeleton disruptor. Biochem Biophys Res Commun. 2005;334:769-78.

54. Mukherjee S, Acharya BR, Bhattacharyya B, et al. Genistein arrests cell cycle progression of A549 cells at the G (2)/M phase and depolymerizes interphase microtubules through binding to a unique site of tubulin. Biochemistry. 2010;49:1702-12.

55. Eastell R, O'Neill TW, Hofbauer LC, Langdahl B, Reid IR, Gold DT, et al. Postmenopausal osteoporosis. Nat Rev Dis Primers. 2016;2:16069.

56. Cheng S, Sipilä S, Taaffe DR, et al. Change in bone mass distribution induced by hormone replacement therapy and high-impact physical exercise in post-menopausal women. Bone. 2002;31: 126-35.

57. Kohrt WM, Snead DB, Slatopolsky E, et al. Additive effects of weight-bearing exercise and estrogen on bone mineral density in older women. J Bone Miner Res. 1995;10:1303-11.

58. Wang YX, Li M, Zhang HQ, Tang MX, et al. Opposite function of $\mathrm{ER} \alpha$ and $\mathrm{ER} \beta$ in controlling $17 \beta$-estradiol-mediated osteogenesis in osteoblasts. Arch Med Res. 2016;47:255-61.

$59 . \bullet$ Deepak V, Kayastha P, McNamara LM. Estrogen deficiency attenuates fluid flow-induced [Ca2+]i oscillations and mechanoresponsiveness of MLO-Y4 osteocytes. FASEB J. 2017; https://doi.org/10.1096/fj.201601280R. Paper demonstrating the interaction between estrogen deprivation and mechanical responses of mouse osteocyte-like cells in vitro.

60. Aguirre JI, Plotkin LI, Gortazar AR, Millan MM, O'Brien CA, Manolagas SC, et al. A novel ligand-independent function of the 
estrogen receptor is essential for osteocyte and osteoblast mechanotransduction. J Biol Chem. 2007;282:25501-8.

61. Frost HM. Bone 'mass' and the 'mechanostat': a proposal. Anat Rec. 1987;219:1-9.

62. Lee K, Jessop H, Suswillo R, Zaman G, Lanyon L. Endocrinology: bone adaptation requires oestrogen receptor- $\alpha$. Nature. 2003;424: 389.

63. Abdi F, Alimoradi Z, Haqi P, Mahdizad F. Effects of phytoestrogens on bone mineral density during the menopause transition: a systematic review of randomized, controlled trials. Climacteric. 2016;19: 535-45.

64. Chen X, Garner SC, Quarles LD, Anderson JJ. Effects of genistein on expression of bone markers during MC3T3-E1 osteoblastic cell differentiation. J Nutr Biochem. 2003;14:342-9.

65. Bouillon R, Carmeliet G, Verlinden L, et al. Vitamin D and human health: lessons from vitamin D receptor null mice. Endocr Rev. 2008;29:726-76.

66. Khanal R, Nemere I. Membrane receptors for vitamin D metabolites. Crit Rev Eukaryot Gene Expr. 2007;17:31-47.

67. Willems HM, van den Heuvel EG, Carmeliet G, Schaafsma A, Klein-Nulend J, Bakker AD. VDR dependent and independent effects of 1,25-dihydroxyvitamin D3 on nitric oxide production by osteoblasts. Steroids. 2012;77:126-31.

68. Polymeris AD, Doumouchtsis KK, Giagourta I, et al. Effect of an oral glucose load on PTH, 250HD3, calcium, and phosphorus homeostasis in postmenopausal women. Endocr Res. 2011;36:45-52.

69. Bouillon R, Van Cromphaut S, Carmeliet G. Intestinal calcium absorption: molecular vitamin D mediated mechanisms. J Cell Biochem. 2003;88:332-9.

70. Bakker AD, Joldersma M, Klein-Nulend J, Burger EH. Interactive effects of PTH and mechanical stress on nitric oxide and PGE2 production by primary mouse osteoblastic cells. Am J Physiol Endocrinol Metab. 2003;285:E608-13.

71. Tazawa K, Hoshi K, Kawamoto S, Tanaka M, Ejiri S, Ozawa H. Osteocytic osteolysis observed in rats to which parathyroid hormone was continuously administered. J Bone Miner Metab. 2004;22:524-9.

72. Stern AR, Nicolella DP. Measurement and estimation of osteocyte mechanical strain. Bone. 2013;54:191-5.

73. Beulens JW, Booth SL, van den Heuvel EG, Stoecklin E, Baka A, Vermeer $\mathrm{C}$. The role of menaquinones $\left(\right.$ vitamin $\mathrm{K}_{2}$ ) in human health. Br J Nutr. 2013;110:1357-68.

74. Filip R, Possemiers S, Heyerick A, Pinheiro I, Raszewski G, Davicco MJ, et al. Twelve-month consumption of a polyphenol extract from olive (Olea europaea) in a double blind, randomized trial increases serum total osteocalcin levels and improves serum lipid profiles in postmenopausal women with osteopenia. J Nutr Health Aging. 2015;19:77-86. https://doi.org/10.1007/s12603014-0480-x.

75. Seitz S, Rendenbach C, Barvencik F, Streichert T, Jeschke A, Schulze J, et al. Retinol deprivation partially rescues the skeletal mineralization defects of Phex-deficient Hyp mice. Bone. 2013;53: 231-8. https://doi.org/10.1016/j.bone.2012.12.009.

76. Turner CH, Takano Y, Owan I, et al. Nitric oxide inhibitor LNAME suppresses mechanically induced bone formation in rats. Am J Phys. 1996;270:E634-9.

77. Tan SD, de Vries TJ, Kuijpers-Jagtman AM, Semeins CM, Everts V, Klein-Nulend J. Osteocytes subjected to fluid flow inhibit osteoclast formation and bone resorption. Bone. 2007;41:745-51.

78. Wimalawansa SJ. Nitric oxide and bone. Ann N Y Acad Sci. 2010;1192:391-403.

79. Torricelli P, Fini M, Giavaresi G, et al. L-arginine and L-lysine stimulation on cultured human osteoblasts. Biomed Pharmacother. 2002;56:492-7.
80. Visioli F, Bellomo G, Galli C. Free radical-scavenging properties of olive oil polyphenols. Biochem Biophys Res Commun. 1998;247: $60-4$.

81. Smith EL, Garavito RM, De Witt DL. Prostaglandin endoperoxide H synthases (cyclooxygenases)-1 and -2. J Biol Chem. 1996;271: 33157-60.

82. Procopio A, Alcaro S, Nardi M, et al. Synthesis, biological evaluation, and molecular modeling of oleuropein and its semisynthetic derivatives as cyclooxygenase inhibitors. J Agric Food Chem. 2009;57:11161-7.

83. Shan J, Fu J, Zhao Z, et al. Chlorogenic acid inhibits lipopolysaccharide-induced cyclooxygenase- 2 expression in RAW264.7 cells through suppressing NF-kappaB and JNK/AP-1 activation. Int Immunopharmacol. 2009;9:1042-8.

84. Canalis E, Hott M, Deloffre P, et al. The divalent strontium salt S12911 enhances bone cell replication and bone formation in vitro. Bone. 1996;18:517-23.

85. Bakker AD, Zandieh-Doulabi B, Klein-Nulend J. Strontium ranelate affects signaling from mechanically-stimulated osteocytes towards osteoclasts and osteoblasts. Bone. 2013;53:112-9. https:// doi.org/10.1016/j.bone.2012.11.044.

86. Xiong J, Piemontese M, Thostenson JD, Weinstein RS, Manolagas $\mathrm{SC}, \mathrm{O}$ 'Brien CA. Osteocyte-derived RANKL is a critical mediator of the increased bone resorption caused by dietary calcium deficiency. Bone. 2014;66:146-54. Paper demonstrating the crucial role of osteocyte-derived RANKL for mediating the catabolic effect of calcium deficiency for bone.

87. Xiong J, Onal M, Jilka RL, Weinstein RS, Manolagas SC, O'Brien CA. Matrix-embedded cells control osteoclast formation. Nat Med. 2011;17:1235-41.

88. Wright HL, McCarthy HS, Middleton J, et al. RANK, RANKL and osteoprotegerin in bone biology and disease. Curr Rev Musculoskelet Med. 2009;2:56-64.

89. Tanaka S. Signaling axis in osteoclast biology and therapeutic targeting in the RANKL/RANK/OPG system. Am J Nephrol. 2007;27:466-78.

90. Kennedy OD, Herman BC, Laudier DM, et al. Activation of resorption in fatigue-loaded bone involves both apoptosis and active proosteoclastogenic signaling by distinct osteocyte populations. Bone. 2012;50:1115-22.

91.• Cheung WY, Fritton JC, Morgan SA, et al. Pannexin-1 and P2X7receptor are required for apoptotic osteocytes in fatigued bone to trigger RANKL production in neighboring bystander Osteocytes. J Bone Miner Res. 2016;31:890-9. This paper elucidates the mechanism by which apoptotic osteocytes in an area of fatigue loaded bone trigger RANKL expression and osteoclastogenesis.

92. Shearer MJ, Bolton-Smith C. The U.K. food database for vitamin K and why we need it. Food Chem. 2000;68:213-8.

93. Schurgers LJ, Vermeer C. Determination of phylloquinone and menaquinones in food: effect of food matrix on circulating vitamin K concentrations. Haemostasis. 2000;30:298-307.

94. Iwamoto J, Takeda T, Sato Y, et al. Synergistic effect of vitamin K2 and prostaglandin E2 on cancellous bone mass in hypophysectomized young rats. Calcif Tissue Int. 2006;79:318-25.

95. Hiruma Y, Nakahama K, Fujita H, et al. Vitamin K2 and geranylgeraniol, its side chain component, inhibited osteoclast formation in a different manner. Biochem Biophys Res Commun. 2004:314:24-30.

96. Bakker AD, Klein-Nulend J, Tanck E, Heyligers IC, Albers GH, Lips P, et al. Different responsiveness to mechanical stress of bone cells from osteoporotic versus osteoarthritic donors. Osteoporos Int. 2006;17:827-33. 